\title{
Confirmation of epidural catheter placement in a quadriplegic patient using a nerve stimulator
}

\author{
Sheila Riazi, MD • Ahtsham U. Niazi, MBBS • \\ Paul S. Tumber, MD · Philip Peng, MBBS
}

Received: 2 October 2009/ Accepted: 23 November 2009/Published online: 20 January 2010

(C) Canadian Anesthesiologists' Society 2010

\section{To the Editor,}

Autonomic hyperreflexia (AH) may occur in up to $85 \%$ of patients with spinal lesions above the spinal level of T6 and manifests itself as marked hypertension, bradycardia, headache, and sweating. The triggers of $\mathrm{AH}$ include distention of the bladder, rectum, or cervix, and surgical stimulation below the level of lesion. The use of epidural to treat $\mathrm{AH}$ in patients with spinal cord injury has been described. ${ }^{1}$ However, as assessment of motor and sensory loss is difficult to elicit in a patient with spinal cord injury, the correct placement of the epidural catheter can be challenging. In 1998, Tsui et al. described low current electrical stimulation (1-10 mA) to confirm epidural catheter placement. ${ }^{2}$ This test has shown to have a sensitivity and specificity of $100 \%$ and $91.6 \%$, respectively. We describe a case in which the placement of an epidural catheter in a patient with spinal cord injury was confirmed using a nerve stimulator. The authors received the patient's written permission to report the case.

Following a fall, a 30-yr-old female sustained a traumatic complete spinal injury at the level of $\mathrm{C} 4$. As a result of this injury, she was quadriplegic with sensory and motor levels of C4 and C6, respectively. Four years later, she presented to our hospital for ileal vesicostomy and creation of a pubovaginal sling. She had had a few episodes of AH triggered by bladder catheter blockage. Due to the intra and postoperative risks of $\mathrm{AH}$, it was agreed that the patient would receive a combined thoracic epidural and general anesthesia. On the day of surgery, the patient's skin was

S. Riazi, MD · A. U. Niazi, MBBS ( $\square)$.

P. S. Tumber, MD - P. Peng, MBBS

Toronto Western Hospital, University Health Network,

Toronto, ON, Canada

e-mail: ahtsham.niazi@uhn.on.ca prepped while she was placed in the right lateral position, and the epidural space was identified in the midline at the T9-T10 level with a 17G Tuohy needle. An Arrow ${ }^{\mathrm{TM}}$ Flexitip $^{\text {TM }}$ epidural catheter (Arrow International Inc, Reading, PA, USA) was threaded easily and secured to the skin at $10 \mathrm{~cm}$. A nerve stimulator (Tracer® II Digital Nerve Locator) (Life-Tech, Inc. Stafford, TX, USA) cathode lead was connected to the epidural catheter via an electrocardiogram adapter (Arrow-Johans ${ }^{\mathrm{TM}}$ ECG adapter) (Arrow International Inc, Reading, PA, USA). The anode terminal of the stimulator was connected to an electrode over the patient's left deltoid muscle. The epidural catheter was then primed with sterile normal saline $1 \mathrm{~mL}$. With a frequency of one $\mathrm{Hz}$ with a pulse width of $200 \mathrm{msec}$, the current output was gradually increased from zero until motor activity was visible. At $5 \mathrm{~mA}$, bilateral abdominal wall muscle twitches were observed between the level of the umbilicus and the costal margin, therefore confirming epidural placement of the catheter. At no time did the patient experience any sensation. A test dose of lidocaine $3 \mathrm{~mL}$ with $1 / 200,000$ epinephrine was administered to rule out intravascular placement. General anesthesia was then induced with fentanyl, propofol, and rocuronium, and after the airway was secured and the patient's lungs were ventilated, anesthesia was maintained with sevoflurane, air/oxygen, and a remifentanil infusion $0.02-0.05 \mu \mathrm{g} \cdot \mathrm{kg}^{-1} \cdot \mathrm{min}^{-1}$. During the three-hour operation, $0.25 \%$ bupivacaine $14 \mathrm{~mL}$ was administered into the epidural space, and her blood pressure did not exceed 120/80 mmHg. The patient's trachea was extubated at the end of the procedure. With patient consent, a low thoracic spine $\mathrm{x}$-ray was taken pre and post Omnipaque ${ }^{\circledR}$ contrast dye $(1.5 \mathrm{~mL})$ injection to delineate the final position of the epidural catheter tip (Figure 1). The patient was managed in the intensive care unit overnight. She received $0.2 \%$ ropivacaine $4 \mathrm{~mL} \cdot \mathrm{hr}^{-1}$ via the epidural catheter, and 
Fig. 1 Spine x-ray after injection of contrast dye through the epidural catheter showing dye in the epidural space in lateral (a) and antero-posterior (b) projections confirming correct placement of the epidural catheter
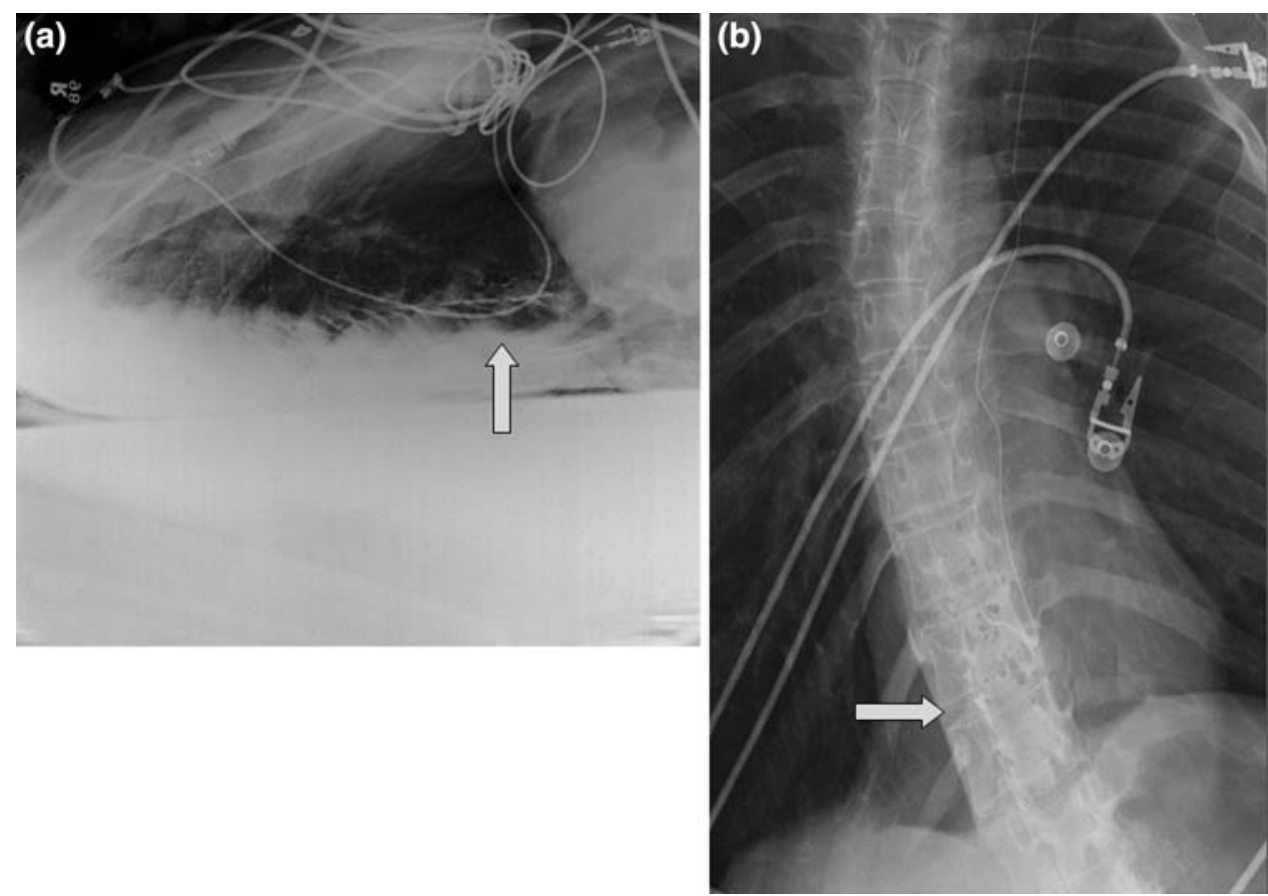

the infusion was discontinued two days postoperatively. She did not experience an episode of AH during her admission.

In the past, the level of epidural anesthesia in quadriplegic patients was confirmed either by conversion of spastic paresis to flaccid paresis ${ }^{3}$ or by hypotension. ${ }^{1}$ In this quadriplegic patient with no spasticity and a low baseline blood pressure, there was no surrogate marker to reliably confirm the position of the epidural catheter; therefore, we decided to use the electrical nerve stimulation method. ${ }^{2}$ Additionally, we used epidurography as an objective method to confirm epidural catheter placement. Patients with high spinal cord injury may have denervation or reduced innervation to abdominal muscles resulting in muscle atrophy. However, as they may have intact lower motor neurons, ${ }^{4}$ neuraxial electrical stimulation can still result in contraction of correlated muscles; nevertheless, higher stimulation currents are to be expected.

Considering that this quadriplegic patient was at perioperative risk of autonomic hyperreflexia, and would therefore benefit from neuraxial blockade, we believe that use of an epidural nerve stimulator provided an objective, reliable, and easy method of confirming epidural catheter placement.

Competing interests None declared.

\section{References}

1. Murphy DB, McGuire G, Peng P. Treatment of autonomic hyperreflexia in a quadriplegic patient by epidural anesthesia in the postoperative period. Anesth Analg 1999; 89: 148-9.

2. Tsui BC, Gupta S, Finucane B. Confirmation of epidural catheter placement using nerve stimulation. Can J Anaesth 1998; 45: 640-4.

3. Stirt JA, Marco A, Conklin KA. Obstetric anesthesia for a quadriplegic patient with autonomic hyperreflexia. Anesthesiology 1979; 51: 560-2.

4. Mulcahey MJ, Smith BT, Betz RR. Evaluation of the lower motor neuron integrity of upper extremity muscles in high level spinal cord injury. Spinal Cord 1999; 37: 585-91. 